\title{
Diseño de edificios de UPIBI -IPN para lograr la
} sustentabilidad

\section{UPIBI design buildings to achieve sustainability -IPN}

\begin{abstract}
Ana Isabel García Monroy
Unidad Profesional Interdisciplinaria de Biotecnología-Instituto Politécnico Nacional,

México

agarciamo@ipn.mx

Lucero Martínez Allende

Unidad Profesional Interdisciplinaria de Biotecnología-Instituto Politécnico Nacional,

México

lumartinez@ipn.mx

Enrique Hernández García

Unidad Profesional Interdisciplinaria de Biotecnología-Instituto Politécnico Nacional,

México

enriquehg21@gmail.com
\end{abstract}

\section{Resumen}

Este proyecto promueve el hacer conciencia a enfocarse, en un cambio rumbo a la sustentabilidad. A su vez, se espera que los alcances del proyecto rebasen las expectativas, para que en los lugares a los que llegue esta iniciativa, puedan comprender los beneficios de realizar un cambio a favor del medio ambiente. En la Unidad Profesional Interdisciplinaria de Biotecnología del Instituto Politécnico Nacional. Se busca revertir el efecto del diseño, construcción y operación el inmueble mediante la implementación, de techos verdes, muros verdes, captación de agua de lluvia .Colocación de cargadores solares para dispositivos electrónicos, Reciclaje Integral. Removiendo elementos que poseen riesgo de contaminación a los ocupantes del edificio y actualizar componentes que pudieran mejorar su eficiencia. Basándose en la arquitectura sustentable recomendados por organismos de certificación de edificios. Para lograr una certificación LEED

Palabras claves: Sustentabilidad, muros, losas, LEED 


\section{Abstract}

This project promotes awareness to focus on a change towards sustainability. In turn, it is expected that the scope of the project exceeded expectations, so that in places it reaches this initiative, to understand the benefits of making a change for the environment. In the Professional Interdisciplinary Biotechnology Unit of the National Polytechnic Institute. It seeks to reverse the effect of the design, construction and operation of the property through the implementation of green roofs, green walls, rainwater catchment .Colocación solar chargers for electronic devices, Integral Recycling. Removing elements with risk of contamination to building occupants and upgrade components that would improve efficiency. Based on sustainable architecture recommended by certification of buildings. To achieve LEED certificationKeywords: Sustainability, walls, slabs, LEED

Key words: Sustainability, walls, slabs, LEED.

Fecha Recepción: Mayo 2015 Fecha Aceptación: Noviembre 2015

\section{Introducción}

Este proyecto promueve el hacer conciencia a enfocarse, en un cambio rumbo a la sustentabilidad. A su vez, se espera que los alcances del proyecto rebasen las Expectativas, esto para que en los lugares a los que llegue esta iniciativa, puedan Comprender los beneficios de realizar un cambio a favor del medio ambiente, donde el desarrollo sustentable es el camino para alcanzar una economía caracterizada por la prosperidad, equidad y un ambiente saludable, como un marco para integrar los objetivos económicos, sociales y ambientales. La sustentabilidad "Es la capacidad de satisfacer necesidades de la generación humana actual sin que esto suponga la anulación de que las generaciones futuras también puedas satisfacer las necesidades propias.” Christopher Jenks. 
El proyecto tiene tres vertientes trascendentales para lograr la sustentabilidad

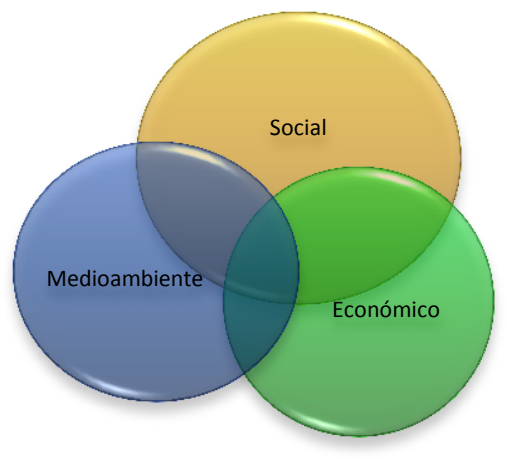

Imagen 1. Objetivo General: Diseño de estrategias para lograr la sustentabilidad en UPIBI-IPN

\section{Marco teórico}

El Programa de Acción para el Desarrollo Sustentable suscrito durante la Cumbre de la Tierra en Río de Janeiro, México (1992). Se comprometió a adoptar medidas nacionales y globales en materia de sustentabilidad, como también acciones orientadas a la generación de indicadores a través de los cuales se puedan medir y evaluar las políticas y estrategias de desarrollo sustentable (INE, 2002). La Comisión de Desarrollo Sustentable (CDS) de las Naciones Unidas aprobó el Programa de Trabajo sobre Indicadores de Desarrollo Sustentable 1995-2000. México comenzó su participación de manera informal.

En 1997 el INE dio a conocer el documento "Avances en el Desarrollo de Indicadores para la Evaluación del Desempeño Ambiental en México" en el cual se presentan indicadores para los temas de calidad del aire, residuos peligrosos, residuos sólidos municipales, vida silvestre, áreas naturales protegidas, cambio climático y disminución del ozono estratosférico. Aplicado al diseño y la arquitectura, estos adjetivos se integran en construcciones que designan los edificios que se construyan y funcionen de acuerdo a los condicionantes y posibilidades ambientales del lugar (clima, valores ecológicos), sus habitantes y modos de vida. 
Esto se logra mediante dos subsistemas: el de conservación y uso racional de la energía y el de los sistemas solares pasivos, incorporados ambos al organismo arquitectónico. Por extensión se aplican al urbanismo. (INE, 2004).

\section{Arquitectura sustentable}

La Arquitectura sustentable contempla cinco principios medulares; esencialmente: Obtener el mayor rendimiento con el menor impacto al ecosistema de construcción. Lograr una eeficacia y moderación en el uso de materiales de construcción. Tener una reducción de cantidad del consumo de energía, procurando utilizar fuentes de energía renovable. Minimizando el balance energético global de la edificación. Cumpliendo los requisitos de confort, salubridad, iluminación y habitabilidad del edificio.

La arquitectura sustentable; se divide a su vez en cinco rubros: Ahorro de energía. Implantación y emplazamiento. Materiales para edificios sustentables. Manejo de residuos. Reciclado de estructuras y materiales

El sistema de certificación LEED (Leadership in Energy and Environmental Design).

Es un estándar con referencia en edificación sostenible a nivel mundial, otorgada por el USBGC, aceptada como emblema en cuanto al diseño, edificación y gestión de edificios sostenibles y eco eficientes. Consiste en una certificación otorgada a un edificio nuevo o existente con base en un sistema de puntuación que evalúa la eficiencia energética, el uso de energías limpias y renovables, el aprovechamiento y gestión del agua, los materiales empleados, el entorno y el ambiente interior.

\section{Techos Verdes}

Los techos verdes es una forma de aprovechar los espacios, en los edificios. Siendo capaces de retener hasta el $90 \%$ de la precipitación. Una gran parte de esta agua es devuelta a la atmósfera, el resto fluye de forma retardada a los sistemas de desagüe, así se puede disminuir la dimensión de los conductos y a la vez se reduce el desagüe. Reduciendo el calentamiento atmosférico y humedecen el ambiente urbano creando así un clima más agradable. Actuando como filtro que retiene elementos tóxicos, por lo que contribuyen a 
reducir la contaminación atmosférica. Del mismo modo, el sustrato filtra el agua de la lluvia reduciendo en ella las sustancias nocivas, como el ruido reduciendo la reflexión sonora hasta 3DB y son capaces de mejorar la insonorización hasta 8DB.

\section{NORMATIVIDAD}

Para desarrollar un sistema en un edificio, se necesita un eje rector como lo es una norma pertinente. Esta norma expedida por el Gobierno de la República a través de la SEMARNAT, funge como un punto clave en la viabilidad de un sistema.

En 2008 el Gobierno del Distrito Federal (GDF) puso en marcha el aún llamado Programa de Certificación de Edificaciones Sustentables (PCES), que pretende establecer un estándar para calificar los edificios tanto habitacionales como comerciales y ofrecer así una serie de incentivos fiscales, que van desde descuentos en el impuesto predial y licencias de construcción hasta financiamientos a tasas preferenciales y rapidez en la ejecución de trámites.

NMX-AA-164-SCF1-2013 de Edificación Sustentable

La NADF-013-RNAT-2007, especifica detalladamente cuales son las especificaciones, componentes y requerimientos mínimos que debe cumplir la ejecución de una cubierta verde "Que estableciendo las especificaciones técnicas para la instalación de sistemas en el Distrito Federal"

\section{Lombricomposta}

La lombricomposta es un método cada vez más popular de composteo pasivo y se reconoce como el composteo del futuro.Para elaborar la lombricomposta se introduce la lombriz roja "lombriz californiana". Profesores investigadores de UPIBI trabajan con producción de la lombriz no tendríamos problemas en la obtención de las lombrices $\mathrm{Si}$ creamos las condiciones óptimas para que se desarrollen las lombrices, ellos nos pueden elaborar un humus/ abono de excelente calidad sin que nosotros tenemos que hacer el trabajo de hacer las pilas y traspalear. 


\section{Desarrollo}

En principio hay que tomar en cuenta que una Escuela es un sistema con un flujo entrante como se muestra en la Foto 1:

- Electricidad.

- Gas natural o LP

- Agua

- Insumos

- materiales.

- alimentos.

- equipo.

- Etc

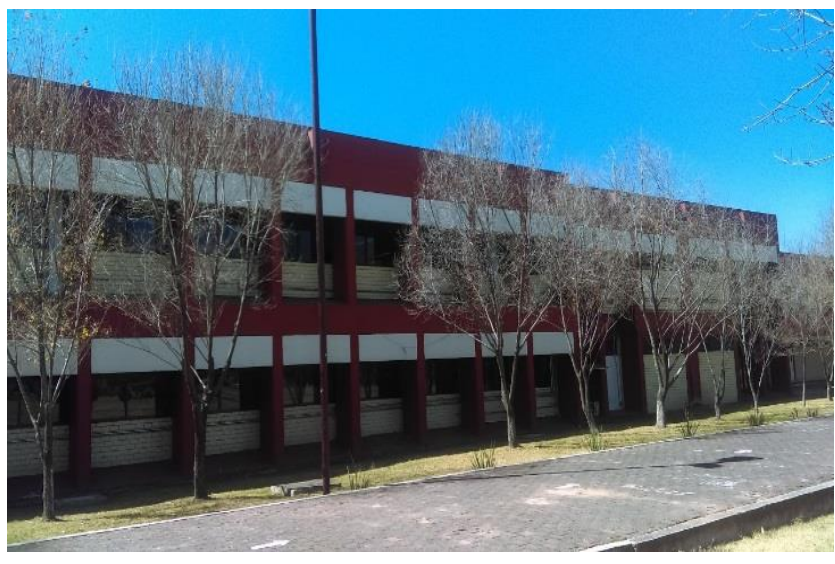

Foto 1. Edificio de UPIBI

Flujo saliente

- Aguas grises y negras

- Residuos orgánicos Biodegradables

- Residuos Solidos

- Residuos peligrosos

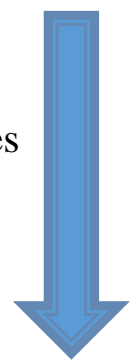

\section{Los Pasos a seguir en el Proyecto de Sustentabilidad en UPIBI-IPN}

1.- Tener una visión de futuro a largo plazo para identificar los rumbos. 2.- Medir los resultados de nuestras acciones (indicadores).

3.- capacitación y concientización por parte del comité ambiental 
3.- Considerar la panorámica completa, las escuelas sustentables tienen un enfoque integrar de todas las interrelaciones existentes.

Para poder lograr la sustentabilidad en UPIBI es indispensable realizar un programa de cada una de las acciones a realizar mediante unas propuestas.

$>$ Reciclaje integral

$>$ Composta

$>$ Arquitectura sustentable ( Techos verdes)

$>$ Colocación de cargadores solares para Laptop y Celulares

$>$ Aprovechamiento de agua de lluvias

Requeriremos

$>$ Infraestructura

$>$ Capacitación

$>$ Información

$>$ Cultura Organizacional

\section{Reciclaje Integral}

\section{Propuesta}

El proyecto pretende disminuir el impacto ambiental mediante propuestas

El comité ambiental de UPIBI-IPN tiene trabajando exhaustivamente en informar y explicar la separación de los residuos, pero desafortunadamente no sé a logrado una separación adecuada, logrando ver una problemática en la identificación en los contenedores. Para recolectar los residuos por lo que el Reciclaje Integral es la solución.

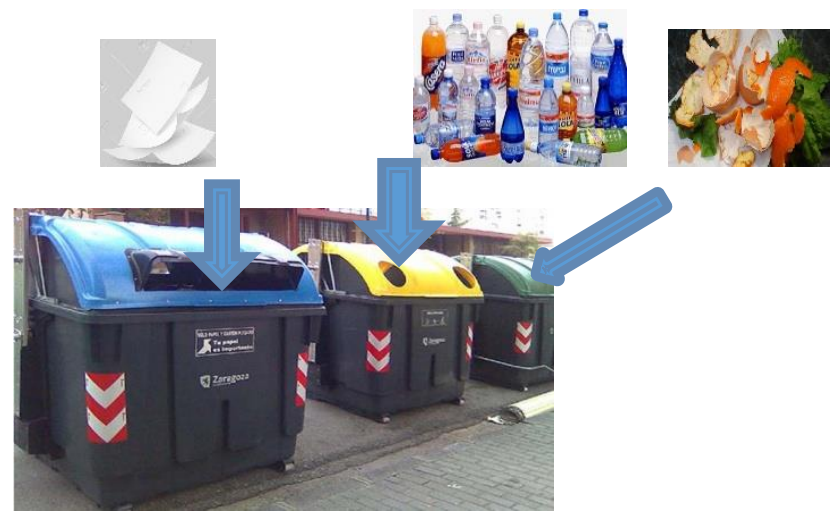

Foto.2 Contenedor tipo 
La propuesta consiste en colocar contenedores que tengan un dispositivo especial como se observa en la Foto 2. Para la colocación adecuada dé los desechos, la recolección será más eficiente. Contamos con estudiantes de Ingeniería Biomédica que cursan las materias de biomateriales y procesos de manufactura. La propuesta consiste en que los alumnos manufacturen los contenedores. El diseño de los contenedores, en su parte de atrás debe ser de fácil acceso para la recolección.

De cartón (venta), Vidrio (venta), Papel (reciclar), Basura orgánica (composta), Metales ( Aluminio - venta), Plástico (Pet Venta), Otros ( Tirar),Peligrosos.

\section{Propuesta}

\section{Composta (Lombricomposta)}

La composta Lombricomposta será empleada en los jardines de la UPIBI. Para la preparación de la composta se emplearan todos los desechos orgánicos generados por cafetería. De esta forma, la aplicación de composta es un beneficio para el suelo. La incineración y el vertido controlado son hasta ahora los tratamientos más habituales de los residuos orgánicos, aunque el compostaje es el único que permite recuperar sus nutrientes e incorporarlos por completo, al ecosistema.

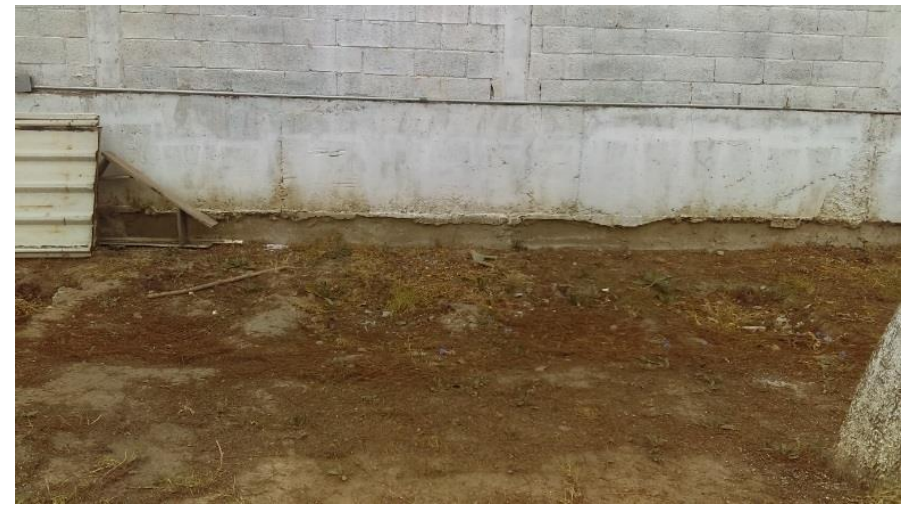

Foto 3. Ubicación de la lombricomposta

En la lombricomposta debemos tener cuidado con el exceso de humedad, pero tampoco deje secar la composta, porque las lombrices dejan de reproducirse y se van. Necesitan una humedad relativa de $70 \%$ en el suelo. La temperatura ideal para que se reproduzcan en de 
21 centígrados. No les gustan las temperaturas elevadas o fríos excesivos. Elegimos el lugar que cumple con las características idóneas como podemos observar en la Foto3 es un rincón protegido y sombreado.

\section{Propuesta}

\section{Arquitectura Sustentabilidad (Techos Verdes en Edificios de UPIBI)}

La propuesta consiste en sembrar árboles que se han secado alrededor del edificio de gobierno. Sembrar hortalizas (Muros Verdes) y techos verdes con la finalidad de motivar al personal de UPIBI.

El riego de los árboles se recomienda realizarlos por las tardes y mañanas para el ahorro del agua.

$>$ Evitar fugas y desperdicios de agua (reportarlo)

$>$ A largo plazo realizar un proyecto de aprovechamiento de las aguas pluviales.

Difusión de estrategias de ahorro de agua

Techos verdes consiste en la la reforestación de diferentes áreas la colocación de elementos que son necesarios que compensan la falta de adhesión de las plantas al suelo natural y asegurar de esta forma el éxito de la vegetación. Los componentes propuestos son seis, estando acoplados entre sí de tal forma que garantizan un funcionamiento seguro y duradero como se observa en la Imagen 2.

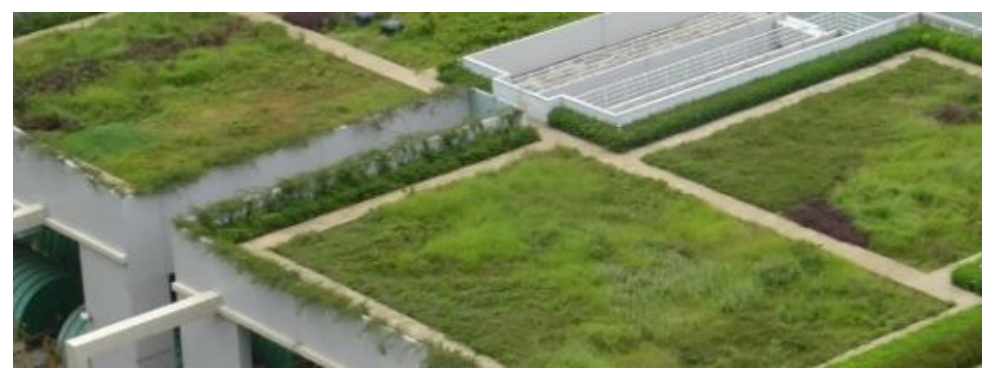

Imagen 2. Techos verdes

En primer lugar tenemos que colocar unas láminas antirraíz que protegen la impermeabilización de los edificios. 
En segundo lugar es la colocación de mantas protectoras de las láminas de antirraízde que evitan un daño por retención de agua y nutrientes de las plantas.

El tercer lugar se colocaría las capas drenantes, cuya función es almacenar agua de la lluvia y del riego en las cavidades superiores de las placas, conduciendo el agua sobrante de manera rápida y segura a los sumideros de la cubierta a través de sus canaletas inferiores. Al tiempo que aseguran una adecuada oxigenación de la tierra de las plantas.

El cuarto punto se coloca una capa filtrante para evitar que los granos finos de la capa adhesiva superior se corroan y asegurar el funcionamiento seguro del drenaje.

En el quinto punto es la colocación del sustrato mineral, que puede ajustarse a las necesidades particulares de la vegetación para garantizar su máximo desarrollo. Como último componente es la plantación de las plantas.

Nuestra propuesta es la implementación de techos verdes en el edificio de gobierno elaborada por alumnos de ingeniería Ambiental, teniendo como ventajas

Retención del agua: Las cubiertas verdes son capaces de retener hasta el $90 \%$ de la precipitación. Una gran parte de esta agua es devuelta a la atmósfera, el resto fluye de forma retardada a los sistemas de desagüe, así se puede disminuir la dimensión de los conductos y a la vez se reduce el desagüe.

Mejora del Clima Urbano: Las cubiertas verdes reducen el calentamiento atmosférico y humedecen el ambiente urbano creando así un clima más agradable.

Reducción de la contaminación: Las cubiertas verdes actúan como filtro que retiene elementos tóxicos, por lo que contribuyen a reducir la contaminación atmosférica. Del mismo modo, el sustrato filtra el agua de la lluvia reduciendo en ella las sustancias nocivas.

Protección contra el Ruido: Las cubiertas verdes reducen la reflexión sonora hasta 3DB y son capaces de mejorar la insonorización hasta 8DB.Para edificios rodeados de focos ruidosos. 


\section{Propuesta}

Colocación de cargadores solares para dispositivos electrónicos (laptops, celulares y tables)

El consumo de energía eléctrica es uno de los factores que afectan en gran medida el medio ambiente. La solución es la utilización de energías renovables (energía solar)

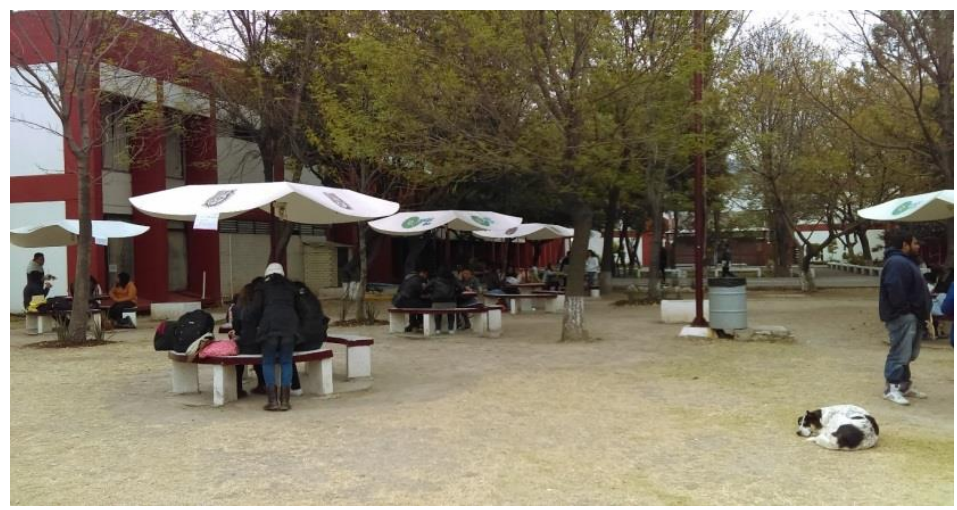

Foto4 distribución de palapas

La propuesta consiste en colocarlos en 11 palapas que no tienen obstáculos de sombra sobre los paneles. Teniendo como ventajas:

$>$ Son sistemas de fácil instalación

$>$ Elevada versatilidad

$>$ Instalación fácilmente modulables

$>$ Costos energéticos nulos

$>$ Mantenimiento bajo

$>$ Es más respetuoso con el medioambiente

\section{Aprovechamiento de agua pluvial}

\section{Propuesta:}

Consiste en el diseño de una cisterna para la captación de aguas pluviales en los edificios. Tiene la finalidad de aprovechar el agua pluvial para los sanitarios y riego de jardines El uso de agua de lluvia 
Recoger agua de lluvia supone una gran ventaja, ya que es bastante limpia, es gratuita y además no se requiere de instalaciones complicadas para ello. Sin utilizar ningún tratamiento, el agua de lluvia puede servir para el inodoro, lavadora, lavavajillas, limpieza del hogar, y riego de jardines. Si se quisiera disponer para el aseo personal, cocinar y beber, entonces se tendría que potabilizar y realizar análisis periódicos para disponer de todas las garantías sanitarias.

Inodoros:

1. Inodoros con cisterna elevada: la cisterna está colocada en altura y la descarga esta accionada mediante un tirador.

2. Sistema de doble descarga y de interrupción de descarga pueden ahorrar agua mediante la incorporación de un sistema de descarga que permite escoger entre dos volúmenes distintos de descarga (de 3-4 L).

Urinarios:

1. Con temporizador provisto de un pulsador cuyo accionamiento provoca una abundante descarga de agua.

2. Con célula fotoeléctrica utiliza la tecnología de infrarrojos para detectar la presencia de un usuario.

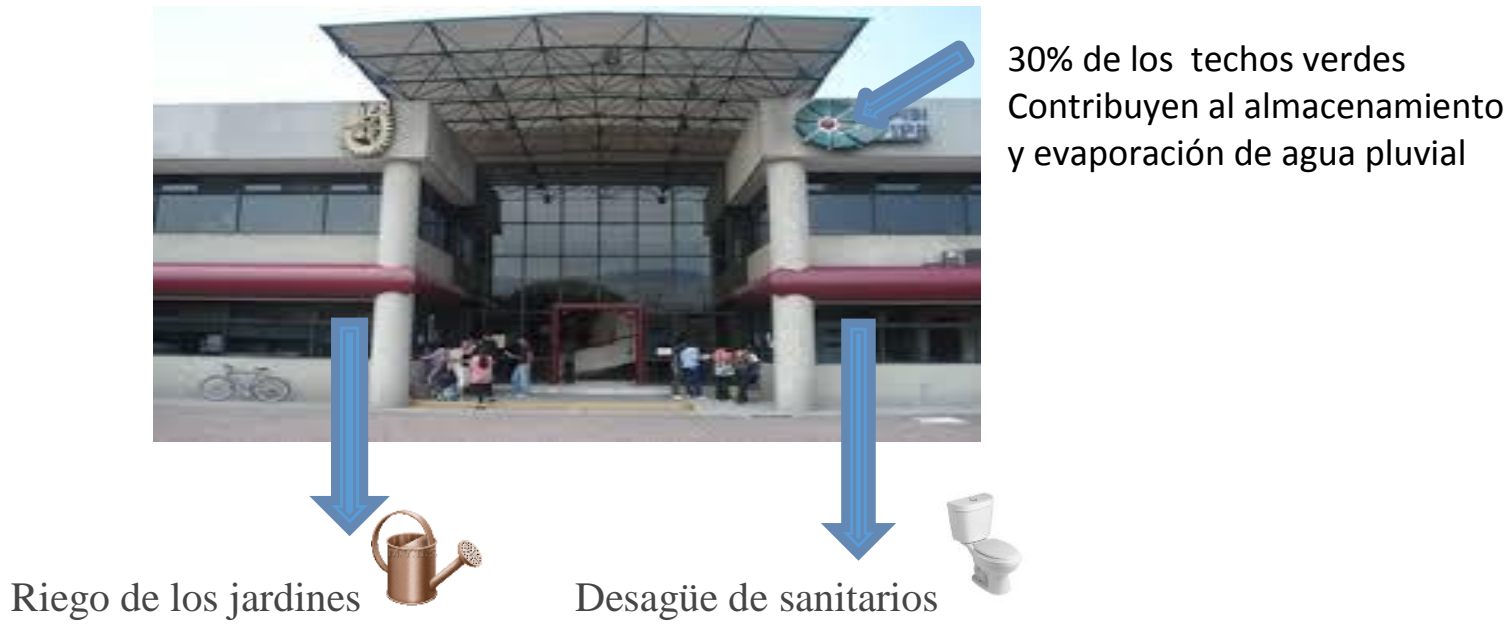

Foto 3 Edificio de gobierno 


\section{Conclusiones}

La sustentabilidad se alcanza mediante la acción sinérgica de diversos elementos básicos en un sistema; en este caso el agua, energía eléctrica, reciclaje integral y materiales. Es trascendental el trabajo colaborativo de todos los integrantes de UPIBI-IPN

Esta transformación busca cambiar la manera de actuar de la comunidad de UPIBI-IPN, trabajando en conjunto sin poner en riesgo el bienestar de las generaciones futuras.

No olvidar que se requiere Capacitación intensiva, Capacitación continua y Monitoreo del reciclado integral.

\section{Bibliografía}

“Gestión y legislación ambiental”.(2007) Instituto Nacional de Ecología (INE). México, D.F. Disponible en: www.ine.gob.mx. Fecha de consulta: mayo de 2015.

“Arquitectura Sustentable". (2004) Disponible en: https://arteyarq.wordpress.com/arquitectura-sustentable/ Fecha de consulta: mayo de 2015

"Sobre el desarrollo sustentable". (2012) Centro de Estudios para el Desarrollo Sustentable (CEDES). Escuela de Asuntos Ambientales. Puerto Rico. Disponible en: http://www.suagm.edu/umet/cedes/pdf/presentacion_des_sust.pdf Fecha de consulta: mayo de 2015

Rodríguez M. L.C. (2010). Certificación Leed e Integración En Edificaciones. Septiembre 30,2014, de Tecno Edificios pp. 19-35

LEED-NC versión 2.2 reference guide, indoor enviromental quality, October 2005, pp 287388

Green Building Academy. Educating for a Greener Destination. Chapter 6- Indoor Environmental Quality. Consultado en: 16/08/14 (Sitio en línea ) http://www.greenbuildingacademy.co/leed-ga/indoor-environmental-quality/ 